\title{
Note sur la sensibilité héréditaire des bovins à la streptothricose
}

\author{
par R. DUMAS (*), P. LHOSTE (*), N. CHABEUF (**), J. BLANCOU (**)
}

\section{RESUME}

\begin{abstract}
Certaines races bovines sont plus sensibles que d'autres à la streptothricose. Une étude statistique de 371 sujets, descendants de 21 taureaux Brahman, démontre que la race est plus réceptive à la maladie que les zébus indigènes du Cameroun et de Madagascar et que cette réceptivité varie avec une différence significative selon les taureaux. Ceci pourrait permettre une sélection des lignées les moins sensibles.
\end{abstract}

\section{INTRODUCTION}

Les différences de réceptivité des animaux aux agents microbiens sont reconnues dans nombre de maladies. Ces différences de réceptivité peuvent être liées à des facteurs extrinsèques (facteurs climatologiques et facteurs d'agressions divers) ou à des facteurs intrinsèques (individu, âge, sexe, race). Ces différences de sensibilité raciales sont exploitées dans certains cas pour obtenir des lignées plus résistantes (ou plus sensibles) à certaines maladies. En ce qui concerne la streptothricose bovine, de telles différences de réceptivité ont été signalées par différents auteurs.

\section{DIFFERENCES DE RECEPTIVITE LIEES A DES FACTEURS EXTRINSEQUES}

\section{Facteurs climatiques}

L'influence de la saison sur le déroulement de la maladie est apparue comme très importante pour la majorité des auteurs qui en ont étudié l'épizootologie: BRAIBANT, BUCK, CHODNICK, PIER, RIBOT, SERRES, SOL-

(") I.E.M.V.T., Centre de recherches zootechniques Wakwa, Cameroun.

(**) I.E.M.V.T., Région de recherches de Madagascar.
TYS $(2,3,5,14,18,19,20)$ signalent la recrudescence et la gravité des cas en saison des pluies et KELLEY (10) a noté que la majorité des cas surviennent en été, aux EtatsUnis. Une étude de laboratoire de MACADAM (11) tend à prouver que l'humidité ne favorise pas les lésions mais au contraire les guérit : cette opinion a été critiquée par HART (8) qui reproche à l'étude expérimentale de ne pas reproduire les lésions naturelles.

L'influence de l'altitude avait été signalée par BUCK (3) qui signale la rareté des cas aux altitudes supérieures à 1.500 mètres.

\section{Facteurs favorisants : maladies microbiennes ou parasitaires, traumatismes}

L'influence des facteurs généraux qui diminuent la résistance des bovins (maladies intercurrentes) a été rapportée par BRAIBANT, MORNET, RIBOT $(2,13,18)$ comme favorisant l'évolution de la streptothricose.

Mais ce sont surtout les facteurs locaux traumatisants pour la peau qui sont incriminés : blessures par les épineux selon ZLOTNIK (22) et surtout par le rostre des ixodes selon BUCK, HOBDAY, MAMMERICKX, MORNET, PLOWRIGHT, SOLTYS $(3,9,12,13,15,20)$. L'action de ces facteurs vulnérants locaux n'est cependant pas indispensable et BRAIBANT 
(2) pense en particulier que le rôle des tiques est nul.

\section{DIFFERENCES DE RECEPTIVITE LIEES A DES FACTEURS INTRINSEQUES}

Parmi les facteurs intrinsèques les plus souvent soulignés il faut noter, par odre d'importance : la race, l'âge et la couleur de la robe.

- La couleur de la robe a fait l'objet d'opinions très divergentes, comme le souligne THIERY (21), ce qui tendrait à faire penser qu'elle n'a aucune influence en soi. Mais Thiery pense que peut-être la pigmentation noire des muqueuses et de la peau rendrait les animaux plus résistants.

- L'âge: Certains auteurs signalent une plus grande réceptivité des jeunes animaux, en particulier ALBISTON, BRAIBANT, EGERTON $(1,2,7)$. Mais ce n'est pas l'opinion de THIERY (21), DUMAS (17) ou RIBOT (18).

- La race: les taurins sont reconnus comme plus sensibles que les zébus par BUCK (3), mais THIERY (21) a une opinion opposée, et MORNET (13) ne note aucune différence.

Seuls deux auteurs ont, à notre connaissance, étudié ou démontré ces différences de sensibilité liées à la race : COLEMAN (6) et KELLEY (10).

COLEMAN, au Nigéria, estime qu'il existe chez les bovins N'DAMA une résistance héréditaire totale, raciale et non individuelle, à la maladie.

Les femelles ne transmettaient pas ce caractère de résistance. D'autres races importées au Nigéria (Brahman, Santa-gertrudis) sont plus sensibles que le bétail local. L'auteur attire l'attention des responsables sur la maladie qui est "l'un des obstacles les plus redoutables à l'importation de bétail étranger $»$.

KELLEY, au Kansas, étudie une épizootie sévissant parmi plus de 700 jeunes bovins: 31 malades sur 39 étaient de race "Shorthorn" alors que cette population était en minorité dans le troupeau. L'auteur conclut à l'existence d'une sensibilité héréditaire, et exclut l'apparition fortuite d'un gène à un seul locus (porteur de caractère "sensibilité ») par l'étude de l'origine des animaux.

Le but de la présente note est de rapporter les observations faites au Cameroun et à Mada- gascar, sur la sensibilité particulière de la race Brahman.

\section{MATERIEL ET METHODES}

Les observations concernant la sensibilité particulière de la race Brahman portent sur les troupeaux de deux stations zootechniques situées dans des conditions climatiques assez semblables: Station de Wakwa (plateau de l'Adamaoua au Cameroun) et Station de Kianjasoa (plateau du Moyen-Ouest de Madagascar). Dans ces deux pays ont été importés en vue de l'amélioration du bétail local des zébus de race Brahman du Texas.

A Wakwa, il n'a été importé que des mâles (45 sujets, répartis en 5 lots arrivés de 1952 à 1958).

A Kianjasoa ont été importés des mâles et des femelles.

Les conditions d'étude de la maladie ont été un peu différentes dans les deux stations car, à Wakwa, les animaux subissaient uniquement un traitement ixodicide (H.C.H. Lindane) alors qu'à Kianjasoa les animaux étaient traités à la solution Cooper qui contient du crésyl (bactéricide très actif sur Dermatophilus congolensis (17). Mais dans cette dernière station le rythme des bains ayant été ralenti en 1968 - 1969, la maladie y a sévi avec la même intensité qu'à Wakwa entre 1960 et 1964.

Dans les deux stations, les zébus Brahman et leurs produits ont été rapidement reconnus comme plus sensibles que les zébus de race locale.

Cette sensibilité a été étudiée, autant que possible, selon les mêmes méthodes dans les deux stations.

\section{ETUDE DE LA SENSIBILITE DE LA RACE BRAHMAN EN GENERAL}

Cette sensibilité est rapportée dans de tableau $\mathrm{n}^{0}$ I qui indique les taux de morbidité de la maladie.

D'après ce tableau la sensibilité paraît bien liée au degré de sang Brahman. Il n'a pas été tenu compte du sexe des animaux, car à 
Retour au menu

TABLEAU $\mathbb{N}^{\circ} \mathrm{I}$

\begin{tabular}{|c|c|c|c|c|}
\hline $\begin{array}{c}\text { Degré de sang } \\
\text { Brahman }\end{array}$ & \multicolumn{4}{|c|}{ Station d'observation } \\
\hline \multirow[b]{3}{*}{ Brahmar pur } & \multicolumn{2}{|c|}{ Cameroun } & \multicolumn{2}{|c|}{ Madagascar } \\
\hline & $\begin{array}{l}\text { Effectif } \\
\text { atteint }\end{array}$ & $\begin{array}{c}\text { Proportion } \\
\text { (p.100) }\end{array}$ & $\begin{array}{l}\text { Effectif } \\
\text { atteint }\end{array}$ & $\begin{array}{l}\text { Proportion } \\
\text { (p.100) }\end{array}$ \\
\hline & $32 / 45$ & 71 & $32 / 61$ & 52,4 \\
\hline 3/4 Brahman & \multicolumn{2}{|c|}{ non abservë } & $70 / 136$ & 51,4 \\
\hline 1/2 Brahman & $332 / 966$ & 34,4 & \multicolumn{2}{|c|}{ non observé } \\
\hline 1/4 Brahman & & 5 & \multicolumn{2}{|c|}{ non observé } \\
\hline Zébu Indigène & & 5 & & 6 \\
\hline
\end{tabular}

TABLEAU $\mathrm{N}^{\circ}$ II

\begin{tabular}{|c|c|c|c|c|c|}
\hline \multirow{2}{*}{$\begin{array}{l}\text { Station } \\
\text { d'étude }\end{array}$} & \multirow{2}{*}{ Lignêe } & \multicolumn{2}{|c|}{$\begin{array}{l}\text { Morbiditê chez les } \\
\text { descendants }\end{array}$} & \multirow{2}{*}{$\begin{array}{l}\text { Sensibilité } \\
\text { de la } \\
\text { lignée } \\
\text { (p.100) }\end{array}$} & \multirow{2}{*}{$\begin{array}{c}\text { Limite de confiance } \\
\text { au risque } \\
5 \text { p. } 100\end{array}$} \\
\hline & & Population & $\begin{array}{l}\text { Nombre de } \\
\text { malades }\end{array}$ & & \\
\hline \multirow{17}{*}{ Cameroun } & 1 & 26 & 4 & 15,5 & $4-35$ p. 100 \\
\hline & 2 & 71 & 12 & 16,9 & $9-27$ \\
\hline & 3 & 18 & 4 & 22,2 & $7-46$ \\
\hline & 4 & 58 & 15 & 25,9 & $16-40$ \\
\hline & 5 & 126 & 33 & 26,2 & $18-36$ \\
\hline & 6 & 19 & 5 & 26,3 & $26-50$ \\
\hline & 7 & 52 & 14 & 26,9 & $15-40$ \\
\hline & $B$ & 106 & 30 & 28,3 & $19-38$ \\
\hline & 9 & 40 & 12 & 30 & $17-47$ \\
\hline & 10 & 90 & 27 & 30 & $21-41$ \\
\hline & 11 & 83 & 32 & 38,5 & $28-50$ \\
\hline & 12 & 47 & 19 & 40,4 & $26-55$ \\
\hline & 13 & 55 & 25 & 45,4 & $31-59$ \\
\hline & 14 & 35 & 16 & 45,7 & $28-63$ \\
\hline & 15 & 24 & 11 & 45,8 & $25-66$ \\
\hline & 16 & 59 & 29 & 49,1 & $37-63$ \\
\hline & 17 & 23 & 14 & 60,9 & $41-81$ \\
\hline \multirow{4}{*}{ Madagascar } & 221 & 48 & 13 & 27 & $16-41$ \\
\hline & 2023 & 15 & 8 & 53,3 & $26-79$ \\
\hline & 496 & 31 & 23 & 74,2 & $56-90$ \\
\hline & 109 & 32 & 24 & 75 & $57-89$ \\
\hline
\end{tabular}


Kianjasoa une étude portant sur 310 malades (133 mâles et 177 femelles) a démontré qu'il n'existait pas de différence statistiquement significative entre les sexes: 51,1 p. 100 des mâles et 42,3 p. 100 des femelles ont été atteints.

\section{ETUDE DE LA SENSIBILITE DES DIFFERENTES LIGNEES BRAHMAN}

Elle est résumée dans le tableau $n^{\circ}$ II qui indique, d'après le taux de morbidité chez les descendants d'un même taureau, la proportion de descendants sensibles.

Pour chaque proportion la limite de confiance, évaluée au risque 5 p. 100 , a été établie. On peut constater que de nombreuses lignées présentent entre elles des différences de sensibilité significatives, ou même hautement significatives: ainsi les lignées 221 et 109 à Madagascar.

\section{CONCLUSION - DISCUSSION}

La sensibilité générale de la race Brahman semble liée à la somme des sensibilités des différentes lignées de cette race.

Il est probable que ce phénomène existe dans de nombreuses autres races. Il doit exister aussi dans la population des zébus indigènes : c'est la mortalité qui frappe régulièrement la fraction sensible de cette population, et la soumet ainsi à une sélection naturelle, qui réduit la sensibilité générale de cette " race". Par ailleurs ce phénomène peut expliquer aussi le rythme cyclique observé dans la maladie naturelle: la fraction sensible de la population ne se reconstitue que progressivement pour arriver à l'âge réceptif.

Si la sensibilité de la race Brahman s'est montrée jusqu'ici comme un sérieux obstacle à sa vulgarisation, une sélection dirigée des géniteurs diffusés en milieu d'élevage traditionnel accélérerait utilement la sélection naturelle chez ces animaux.

\title{
SUMMARY
}

\section{Note on hereditary predisposition of cattle to streptothricosis}

Some breeds are more receptive to streptothricosis than others. A statistic study of 371 animals born from 21 Brahma bulls shows that they are more susceptible to the disease than indigenous cattle in Cameroon and Madagascar, and that some bulls transmit this susceptibility with a highly significant frequency. This fact could allow a selection of less receptive breeders.

\section{RESUMEN}

Nota sobre la sensibilidad hereditaria de los bovinos para con la estreptotricosis

\begin{abstract}
Ciertas razas de bovinos son más sensibles que otras para con la estreptotricosis.

Un estudio estadístico de 371 animales descendientes de 21 toros Brahman demuestra que es más receptiva la dicha raza para con la enfermedad que los cebues indigenos de Camerún y de Madagascar, y que ésta receptividad varia con una diferencia significativa según los toros; lo que podria permitir una selección de las líneas menos sensibles.
\end{abstract}

\section{BIBLIOGRAPHIE}

1. ALBISTON (H. F.), « Mycotic dermatitis in the calf ", Aust. vet. J., 1933, 9: 107.

2. BRAIBANT (E.), «La streptothricose cutanée au Rwanda et au Burundi », Bull. epiz. Dis, Afr., 1962, 10 (4) : 517-21.

3. BUCK (G.), «Actinomycose ou streptothricose cutanée des bovins à Madagascar (Drodro-Boka) ", Bull. Off. int. Epiz., 194829 (2-3): 117-21.

4. BUGYAKI (L.). «Dermatose contagieuse des ruminants et du cheval , Bull. Off. int. Epiz., $1959,51(5-6): 237-51$.
5. CHODNICK (K.S.), \& Mycotic dermatitis of cattle in British West Africa ", J. comp. Path., 1956, 66 : 179-81.

6. COLEMAN (C.M.), «Cutaneous streptothricosis of cattle in West Africa $», V e t$. Riend., 1967, 81 (11) : 251-54.

7. EGERTON (J.R.), "Mycotic dermatitis of cattle », Aust, vet. J., 1964, 40 (11) : 388-89.

8. HART (C. B.). "Streptothricosis of cattle », Vet Rec., 1961, 73 (47) : 1.279-80. 
9. HOBDAY (W. S.), Am. Rep. Dept. Vet. Sert. N. Rhodesia, 1952.

10. KELLEY (D. C.) et al., « Cutaneous streptothricosis in Kansas cattle. Part I».Vet. Med. small. anim. clin., 1964, 59 (1): 73-78.

11. MACADAM (1), \& The effect of humidity on the lesions of streptothricosis ", Vct. Rec., 1961, 73 (42): 1.039-41.

12. MAMMERICKX (M.), "Observations sur la dermatose contagieuse des ruminants au Congo », Am. Soc. Belge. Med. trop., 1961, 41 (2) : 133-44.

13. MORNET (P.), THIERY (G.), « Streptothricose cutanée des bovins », Bull. epiz. Dis. Afr., 1955, 3 (3) : 302-24.

14. PIER (A.), NEAL (F. C.), CYSEWSKI (S. J.), "Cutaneous streptothricosis in Kansas cattle ", J. Am. vet. med. Ass., 1963, 142: 995-1.000.

15. PLOWRIGHT (W), "Cutaneous streptothricosis of cattle. Introduction and epizootiological features in Nigeria ", Vet Rec., 1956, 68 (23) : $350-55$.
16. Rapport du C.R.Z. de Wakwa, I.E.M.V.T, Cameroun 1964-1965.

17. Rapport du Laboratoire Central de l'Elevage à Tananarive, Archives I.E.M.V.T.

18. RIBOT (J.J.), * La streptothricose : à propos de quelques notions épidémiologiques à Madagascar ", Terre Malgache (Université de Madagascar ENSA), 1970 (7) : 195-208.

19. SERRES (H.) et collab., "Le croisement brahman à Madagascar ", Rev. Eler. Méd. vét. Pays trop., 1968, 21 (4) : 519-61.

20. SOLTYS (A. M.), * Cutaneous streptothricosis of cattle in the Sudan $»$, Sudan J, vet. Sci. anim. Husb., 1965, 5 : 20-23.

21. THIERY (G.), MEMERY (G.), « La streptothricose cutanée. IV. Etiologie - Traitement Prophylaxie", Rev. Elev. Med. vét. Pays trop., 1961, 14 (4) : 413-27.

22. ZLOTNIK (I.), «Cutaneous streptothricosis in cattle », Vet. Rec., 1955, 67 (33) : 613-14. 